\section{Pacto de Indicadores da Atenção Básica: instrumento de negociação qualificador do processo de gestão do SUS}

\author{
Indicators` Pact for Basic Care: negotiation \\ tool qualifying the unified health system \\ management process
}

Ministério da Saúde. Secretaria de Atenção à Saúde. Departamento de Atenção Básica 1

1 Esplanada dos Ministérios, Bloco G, 7. ${ }^{\circ}$ andar, sala 751. Brasília, DF, Brasil. CEP: 70.058-900. E-mail: dab@saude.gov.br. Site: www.saude.gov.br.
O processo de descentralização do Sistema Único de Saúde (SUS), impulsionado em 1988 com a implantação do Piso de Atenção Básica (PAB), modificou profundamente a face da gestão das ações e serviços no âmbito do setor saúde no Brasil.

Ao priorizar a implantação do PAB, o Ministério da Saúde (MS) buscou, transformando a lógica de financiamento, impulsionar mudanças no funcionamento do modelo de atenção a saúde, tornando-o mais eficiente, garantindo integralidade no desenvolvimento das ações, contribuindo para o acesso universal e consolidando os vínculos entre a população e os serviços.

Objetivando orientar o processo de avaliação e monitoramento da Atenção Básica no âmbito do SUS, o Ministério da Saúde formulou a proposta de desenvolvimento de pactos de gestão entre as Secretarias Estaduais e Municipais de Saúde e o Ministério da Saúde. O Pacto de Indicadores da Atenção Básica foi, então, concebido como um instrumento nacional de monitoramento das ações e serviços de saúde referentes à atenção básica, sendo instituído pela Portaria GM/MS 3.925 de 1998, que aprovou o "Manual para Organização da Atenção Básica", e a Portaria 476 de 1999, que regulamentou o processo de acompanhamento e avaliação da atenção básica. A partir de então a cada ano vêm sendo publicadas, através de portarias específicas, as orientações para o processo de pactuação e a relação de indicadores a serem pactuados pelos gestores.

O Pacto pretende ser um instrumento formal de negociação entre gestores das três esferas de governo (municipal, estadual e federal) tomando como objeto de negociação metas a serem alcançadas em relação a indicadores de saúde previamente acordados. Ao fim de cada ano, municípios e estados têm seu desempenho avaliado pelo nível federal em função do cumprimento das metas pactuadas.

De 1999 a 2003, período em que foi implementado nacionalmente, o processo de pactuação tem apresentado alterações, tanto com relação à sua forma de condução quanto aos indicadores selecionados para avaliação.

Em 1999, o elenco de indicadores municipais perfazia um total de 41: 24 para todos os municípios e 17 específicos para as áreas do Programa Agentes Comunitários de Saúde (PACS) e Programa Saúde da Família (PSF); em 2000, esta relação foi reduzida para 30 indicadores (14 para todos os municípios e 16 específicos para as áreas do PACS e PSF) e em 2001 foram pactuados no total 17 indicadores para os municípios. Os indicadores estaduais, em geral, reproduziam a relação de indicadores municipais, à exceção do ano de 2001 que apresentou dois indicadores exclusivos para os estados, acrescidos aos 17 que eram comuns a ambos, municípios e estados. Para o ano de 2002, foi definida uma relação de 25 indicadores principais, pactuados por todos os estados e municípios, e uma relação de 22 indicadores complementares, de pactuação não obrigatória. Neste ano de 2003, após discussão interna nas áreas técnicas do Ministério e com representações do CONASS (Conselho Nacional de Secretários Estaduais de Saúde) e do CONASEMS (Conselho Nacional de Secretários Municipais de Saúde), foi aprovado em reunião da Comissão Intergestores Tripartite o elenco de indicadores que passa a ser composto de 20 principais e 14 obrigatórios, perfazendo um total de 34 indicadores a serem pactuados por estados e municípios, tendo em vista a repetição de alguns indicadores que já constavam da Programação Pactuada Integrada da Epidemiologia e Controle de Doenças (PPI - ECD). No Quadro 1 encontram-se relacionados os indicadores constantes do Pacto 2003. 
Indicadores do Pacto da Atenção Básica, 2003.

Indicadores principais

1. Número absoluto de óbitos em menores de um ano de idade

2. Taxa de mortalidade infantil

3. Proporção de nascidos vivos com baixo-peso ao nascer

4. Proporção de óbitos em menores de um ano de idade por causas mal definidas

5. Taxa de internação por infecção respiratória aguda em menores de cinco anos de idade

6. Homogeneidade da cobertura vacinal por tetra valente em menores de um ano de idade

7. Taxa de mortalidade materna

8. Proporção de nascidos vivos de mães com quatro ou mais consultas de pré-natal

9. Proporção de óbitos de mulheres em idade fértil investigados

10. Razão entre exames citopatológicos cérvico-vaginais em mulheres de 25 a 59 anos e a população feminina nesta faixa etária

11. Taxa de internação por acidente vascular cerebral (AVC)

12. Taxa de mortalidade por doenças cerebro-vasculares

13. Taxa de internação por cetoacidose e coma diabético

14. Proporção de abandono do tratamento de tuberculose

15. Proporção de abandono do tratamento de hanseníase

16. Taxa de detecção de casos de hanseníase

17. Cobertura de primeira consulta odontológica

18. Razão entre os procedimentos odontológicos coletivos e a população de 0 a 14 anos

19. Proporção da população coberta pelo Programa Saúde da Família (PSF)

20. Média anual de consultas médicas nas especialidades básicas por habitante

Indicadores complementares

1. Número absoluto de óbitos neonatais

2. Taxa de mortalidade infantil neonatal

3. Taxa de mortalidade em mulheres por câncer de colo do útero

4. Taxa de mortalidade em mulheres por câncer de mama

5. Proporção de nascidos vivos de mães com sete ou mais consultas de pré-natal

6. Taxa de internação por insuficiência cardíaca congestiva (ICC)

7. Taxa de internação por diabetes

8. Taxa de incidência de tuberculose pulmonar positiva

9. Taxa de mortalidade por tuberculose

10. Proporção de cura dos casos novos de hanseníase diagnosticados

11. Taxa de prevalência da hanseníase

12. Proporção de grau de incapacidade I e II registrados no momento do diagnóstico

13. Proporção de exodontias em relação às ações odontológicas básicas individuais

14. Média mensal de visitas domiciliares por família 
Dentre as estratégias utilizadas no processo de definição de indicadores e de pactuação, as oficinas de trabalho têm se destacado, constituindo-se em momentos privilegiados de discussão técnica e política sobre o Pacto e de construção de consensos. Desde 1998, o Ministério vem promovendo a realização de oficinas nacionais, que contam com a participação de representantes das Secretarias Estaduais, dos COSEMS e de áreas técnicas do Ministério da Saúde. Estas oficinas têm-se constituído, talvez, nos momentos mais profícuos de aprofundamento do Pacto, aprimorando-se sua qualidade técnica, através do debate em relação aos indicadores.

A avaliação positiva dos participantes das oficinas, a melhoria da qualidade das notas técnicas, a criação de ferramentas (softwares) de apoio ao processo de pactuação, a reprodução pelos estados da estratégia das oficinas para avaliação regionalizada do Pacto são indicativos de que o processo de pactuação tem efetivamente cumprido o seu papel de mobilização e articulação com vistas ao desenvolvimento de ações voltadas para a melhoria da qualidade da atenção básica. Além disso, é surpreendente verificar como o Pacto tem provocado a utilização dos dados dos sistemas de informação em saúde, o que contribui para a melhoria da qualidade das informações.

Vale ressaltar, entretanto, que o processo de pactuação tem sido implementado de forma bastante diversa em âmbito nacional, apresentando matizes que mesclam as características de dois pólos, os quais podem ser denominados de "burocrático" e "dinamizador" conforme Medicina et al. ${ }^{2}$ e apresentados no Quadro 2.

Quadro 2

Características do processo de pactuação no Brasil

Pacto burocrático

Normativo

Entendido como uma demanda do Ministério da Saúde aos estados e municípios

Pontual

Fragmentado

Sem uma efetiva articulação entre setores estratégicos das secretarias

Sem um efetivo monitoramento pelos gestores

Sem visibilidade perante as instâncias de deliberação e pactuação do Sistema Único de Saúde (SUS)

\section{Pacto dinamizador}

Processo aglutinador de experiências e práticas que fortalecem a implementação do processo de municipalização do SUS

Entendido como um instrumento qualificador do processo de gestão do SUS

Utilizado cotidianamente para monitorar e avaliar ações e serviços de saúde

Construído de forma articulada pelas três instâncias de gestão do sus

Integrado ao processo de trabalho dos setores estratégicos das instâncias de gestão do SUS

Instrumento de monitoramento utilizado pelos gestores

Com visibilidade e credibilidade perante as instâncias de deliberação e pactuação do SUS

Fonte: Medina et al. Avaliação atenção básica. Div Saúde Debate 2000.2 
A categorização apresentada enfatiza os aspectos relacionados ao processo de pactuação em lugar daqueles relacionados aos critérios, indicadores e desempenho dos municípios e estados. O texto assinala que o grande desafio posto é fazer a transição do pólo burocrático para o dinamizador. ${ }^{2}$

As mudanças observadas no processo de pactuação e no elenco de indicadores têm resultado, na verdade, do amadurecimento do próprio processo do Pacto da Atenção Básica, traduzindo, um esforço crescente de buscar indicadores mais representativos e robustos na avaliação da atenção básica. É importante destacar que não se trata de avaliar ações específicas no âmbito da atenção básica, mas sim, de avaliar, através de indicadores específicos, globalmente esse nível de atenção, o que só é possível se o indicador apresentar qualidades para tal. Tais qualidades dizem respeito à sua sinteticidade, facilidade de cálculo, disponibilidade, poder discriminatório e robustez.

\section{Referências}

1. Ministério da Saúde. Secretaria de Políticas de Saúde. Departamento de Atenção Básica. Relatório de gestão: 1999-2002. Brasília (DF): O Ministério; 2002.

2. Medina MG, Aquino R, Carvalho ALB. Avaliação da atenção básica: construindo novas ferramentas para o SUS. Div Saúde Debate 2000; (21): 15-28.

Recebido em 10 de março de 2003

Versão final reapresentada em 23 de abril de 2003

Aprovado em 5 de maio de 2003 